\title{
The future of spinal cord stereotaxy
}

\author{
Nadvornik P, Cierny G, Bernadic M \\ Institute of Pathophysiology, Faculty of Medicine, Comenius University, Bratislava, Slovakia. \\ marian.bernadic@fmed.uniba.sk
}

\begin{abstract}
The spinal cord is an integral part of central nervous system, therefore it can be expected that spinal cord has the same properties as the brain. Movement activity is realized by the activation of individual motoneurons of various spinal cord segments under the influence of analytical function of the spinal cord. When a hypothesis is accepted that the mentioned large volume of spinal cord white matter represents the entire length of neuronal network, an idea can be established that the activated motoneurons project through their reticular processes to this connecting network forming a synthetic picture of this movement and after fluent continuity the entire act of movement. Therefore, neuronal network plays the role of dynamic memory.

The perspective of spinal cord stereotaxy in functional neurosurgery hypothetically enables a recognition and understanding of how brain and spinal cord communicate in movement performance (Fig. 2, Ref. 6). Full Text in PDF www.elis.sk.

Key words: spinal cord, central nervous system, neuronal network, stereotaxy.
\end{abstract}

An overview article by Gabriel and Nashold named History of Spinal Cord Stereotaxy was published 15 years ago (1).

According to the authors, the beginnings of spinal cord stereotaxy can be traced back to the end of the 19th century, when a special device was constructed at the Institute of Physiology in Leipzig. It was possible to attach the device to the spinous processes of the neighbouring two vertebrae and delicate instruments could be inserted into the spinal cord to find out the effect of stimulation and lesion with the location that could be determined using microscope.

Therefore, the fundamentals of future functional stereotaxy were established, however, the contemporary level of functional stereotaxy was achieved in brain surgery. At the same time, it became evident that the main requirements for stereotactic interventions on functional brain structures were both their complete anatomical map in principal planes delineated in universal system of coordinates and stereotactic system making insertion of the surgical device to the selected structures defined by the previously obtained coordinates possible.

The two conditions - stereotactic maps and stereotactic device were perfectly realized in a functional stereotaxy of the brain.

The history of spinal cord stereotaxy provided the evidence that the main preconditions for functional spinal cord stereotaxy remained valid although not realized so far.

Though the native function of the spinal cord is the generation of movements dependent on spinal cord motoneurons, stereo-

Institute of Pathophysiology, Faculty of Medicine, Comenius University, Bratislava, Slovakia

Address for correspondence: $\mathrm{M}$. Bernadic, $\mathrm{MD}, \mathrm{PhD}$, Institute of Pathophysiology, Faculty of Medicine, Comenius University, Spitálska 24, SK81372 Bratislava, Slovakia.

Phone: +421.2 .52965400$ tactic maps of the spinal cord respecting this aspect has not been constructed at this time as well as stereotactic spinal cord device able to target defined spinal cord structure according to stereotactic map. There are two unrealized conditions causing that spinal cord stereotaxy lags behind. This situation was also responsible for falling behind of the physiological idea about the communication between brain and spine that can be influenced by spinal cord stereotaxy also from the point of therapeutic implications. Therefore, the aim of the author of the paper is to bridge the gap in knowledge so that the result may further stimulate the new rise of spinal cord stereotaxy in functional neurosurgery.

\section{Methodology and results}

Because the function of the dorsal horns of spinal cord gray matter (Rexed division into nine zones based on animal experiments on cats) is already well established - with an exception being zone No IX, extending as far as the anterior horns of spinal cord gray matter, our work was focused on this zone with the purpose to supplement the location of motoneurons for individual muscles in different spinal cord segments. Therefore, the pathway for targeted stereotactic surgeries will be opened for patients with muscle spasticity caused by infantile palsy or different forms of dystonia.

The starting point of the work was a 30-year work of Prof. Čierny in the Institute of Anatomy, Comenius University Bratislava (2). The author performed a cutting of individual motor nerve innervating defined muscles in cat model (structure of movements similar to humans) and studied the location of motoneurons in spinal cord segments by means of tygroid degeneration technique. It was possible to map the location of motoneurons in anterior horn of spinal cord extending from C1 to L1 (Fig. 1).

What remained for completion was the definition of the location of motoneurons for the lumbosacral part of spinal cord. This 


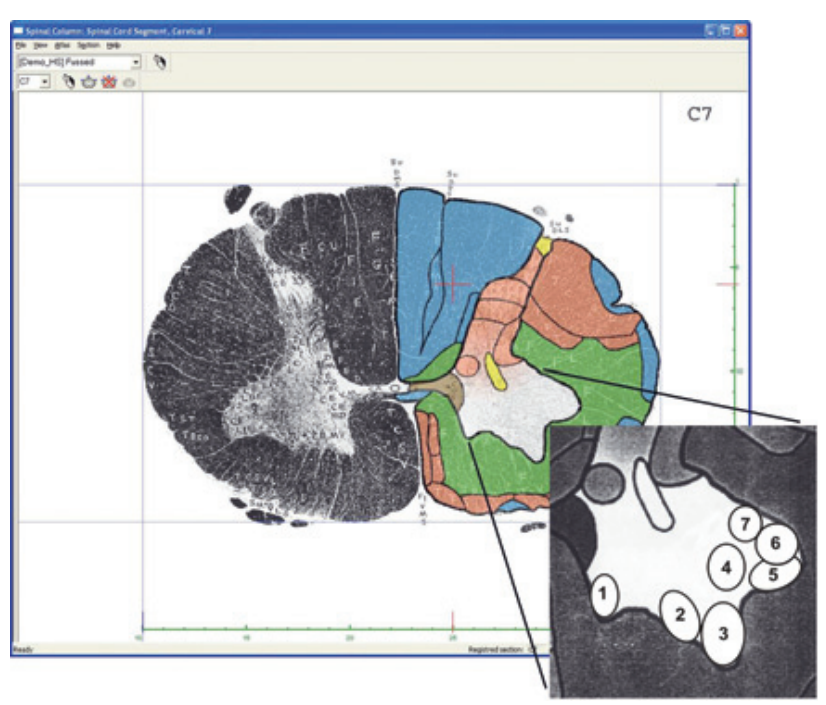

Fig. 1. Spinal cord segment C5 according to Prof. Čierny.

was facilitated by Sharrad's observation (3) who had found the location of motoneurons of the affected muscles in the section material of poliomyelitic patients in the anterior horns of the spinal cord and had made schematic drawings together with detailed marking of their structures with their supposed connection. Riley 's anatomical atlas (transverse sections of all spinal cord segments with detailed description) also became an important source of data (4). However, the author marked only groups of motoneurons without identification in the anterior horns of the spinal cord (2-5 or more when related to spinal cord intumescencies), densely delineated by nerve fibres, with some of them entering the anterior horns of the spinal cord.

The movement performed by the spinal cord is relatively simple, flexion and extension, adduction and abduction, or assistance in pronation and supination. When these particular function were projected into motoneuron location it became evident that the described motoneuron groups reflect muscles providing the functions for individual limb schemes.

Taking into consideration a certain analogy with motoneuron location in cervical and thoracic spinal cord, it was possible to construct the maps of motoneuron location valid for lumbosacral segments and test them during surgeries for spasticity after a complete spine injury at T5 level and hyperspastic bladder at S2,3 level.

The complete stereotactic map of the spinal cord was transformed to computer form to make an automatic calculation of coordinates for location of the selected group of motoneurons possible (6).

However, stereotactic system remains the effector device for a spinal cord stereotaxy. Two requirements were defined for construction: the device can be used for any spinal cord segment between $\mathrm{C} 1$ and L3 and its functional part for surgical device implantation is perpendicular to spinal cord surface. The device was brought into work with the help of Institute of Measurements SAV Bratislava (Fig. 2). It can be attached to any spinous process and

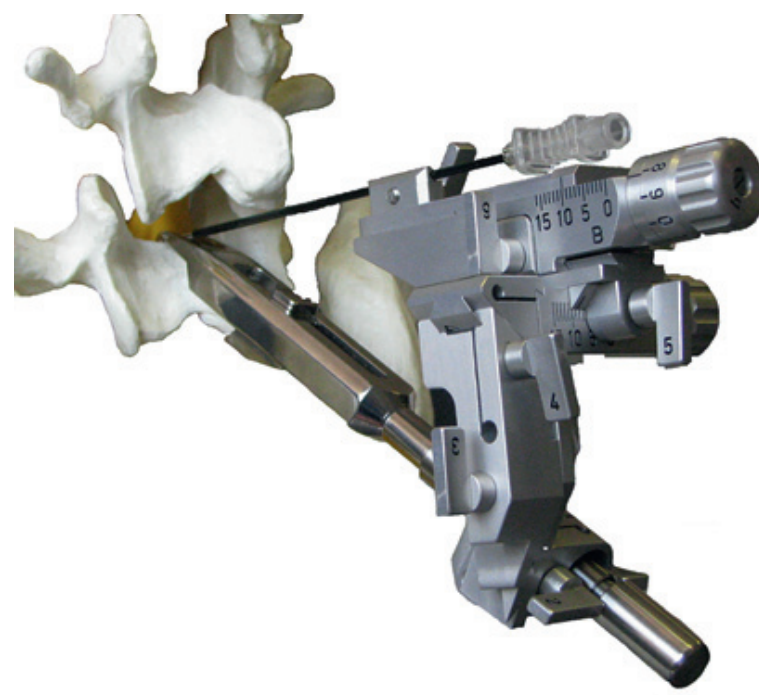

Fig. 2. The universal device for spinal cord stereotaxy.

after dural sac opening the electrode is directed perpendicularly in close proximity of vertebral arch to spinal cord under the control of surgical microscope. The slidding of the electrode is possible in $0.01 \mathrm{~mm}$ steps.

When looking at the cut surface of the spinal cord there is a large space surrounding spinal cord anterior horns between spinal gray matter and the marginal range of white matter containing the known neural pathways connecting brain to the spinal cord.

However, spinal cord is an integral part of central nervous system, therefore it can be expected that spinal cord has the same properties as the brain. Movement activity is realized by the activation of individual motoneurons of various spinal cord segments under the influence of analytical function of the spinal cord. When a hypothesis is accepted that the mentioned large volume of spinal cord white matter represents the entire length of neuronal network, an idea can be established that the activated motoneurons project through their reticular processes to this connecting network forming a synthetic picture of this movement and after fluent continuity the entire act of movement (5). Therefore, neuronal network plays the role of dynamic memory.

The only thing remaining to be explained is how the picture of movement reaches brain memory and what are the neural pathways requesting movement performance from the spinal cord.

With a high probability the task depends on reticulospinal tract passing along the entire length of the spinal cord in close proximity to pyramidal and rubrospinal tract. The experimental stimulation of reticulospinal tract at the level of pontomesencephalic junction elicited a movement reaction despite the fact there was no connection between reticulospinal tract and motor cells (6). The probable explanation is that the nerve tract transmits only movement pictures and that the ascending mechanism of movement communication between brain and spinal cord is acceptable as well as the descending mechanism. 


\section{Conclusions}

Two centuries after the historical birth of spinal cord stereotaxy, the final location of motoneurons for the individual muscles in spinal cord anterior horns has been completed and a complete stereotactic map of all spinal segments was constructed. At the same time, an universal construction of a new stereotactic spinal cord device (surgical parameters can be automatically read from the computer stereotactic map) brings a new stimulus for the development of spinal cord stereotaxy in functional neurosurgery.

Therefore, a possibility was gained to target not only the known stereotactic targets in spinal cord but also the unknown movement related structures, that fulfil the main function of the spinal cord.

The perspective of spinal cord stereotaxy in functional neurosurgery hypothetically enables a recognition and understanding of how brain and spinal cord communicate in movement performance.

\section{References}

1. Gabriel EM, Nashold BS. History of spinal cord stereotaxy. J Neurosurg 1996; 85: 725-731.

2. Čierny G. Motorická lokalizácia v krčnej a hrudnej mieche. Bratislava: Comenius University, 1983: 223.

3. Sharrad JWJ. The distribution of the permanent paralysis in the lower limbs in poliomyelitis. J Bone Jt Surg 1955; 37 B: 540-558.

4. Riley HA. An atlas of the basal ganglia, brain stem and spinal cord. Baltimore:Williams and Wilkins, 1943: 708.

5. Nádvorník P, Čierny G, Bernadič M. Nové poznatky o pravděpodobné součinnosti míchy s mozkem při realizaci pohybu. Čs Slov Neurol N 2009; 72/105: 186-187.

6. Davidson AG, Buford JA, Bilateral action of the reticulospinal tract on arms and shoulder muscles in the monkey: Stimulus triggered averaging. Exp Brain Res 2006; 173: 25-39.

Received February 21, 2012. Accepted January 18, 2013. 